\title{
Solar cycle-related variation in solar differential rotation and meridional flow in solar cycle 24
}

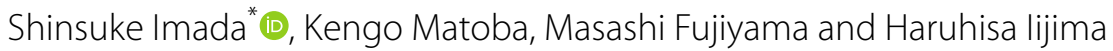

\begin{abstract}
We studied temporal variation of the differential rotation and poleward meridional circulation during solar cycle 24 using the magnetic element feature tracking technique. We used line-of-sight magnetograms obtained using the helioseismic and magnetic imager aboard the Solar Dynamics Observatory from May 01, 2010 to March 26, 2020 (for almost the entire period of solar cycle 24, Carrington rotation from 2096 to 2229) and tracked the magnetic element features every $1 \mathrm{~h}$. We also estimated the differential rotation and poleward meridional flow velocity profiles. The observed profiles are consistent with those of previous studies on different cycles. Typical properties resulting from torsional oscillations can also be observed from solar cycle 24 . The amplitude of the variation was approximately \pm 10 $\mathrm{m} \mathrm{s}^{-1}$. Interestingly, we found that the average meridional flow observed in solar cycle 24 is faster than that observed in solar cycle 23. In particular, during the declining phase of the cycle, the meridional flow of the middle latitude is accelerated from 10 to $17 \mathrm{~m} \mathrm{~s}^{-1}$, which is almost half of the meridional flow itself. The faster meridional flow in solar cycle 24 might be the result of the weakest cycle during the last 100 years.
\end{abstract}

Keywords: Solar surface flow, Solar cycle

\section{Introduction}

The 11-year variations in solar activity are important sources of decadal variations in the solar-terrestrial environment. It is well known that the maximum number of sunspots differs during each 11-year cycle. Some cycles show a large number of sunspots, whereas other cycles show few sunspots (Clette et al. 2014). The prediction of the amplitude of the 11-year sunspot cycle is an important task for the long-term prediction of space weather (Pesnell 2016) because the maximum number of sunspots is closely related to solar flares and coronal mass ejections (e.g., Tsuneta et al. 1992; Imada et al. 2007, 2011, 2013). The solar cycle 24 was a peculiar cycle with the fewest sunspots observed during the last 100 years (Svalgaard et al. 2005). However, we still do not understand why solar cycle 24 has been weak and

*Correspondence: imada@nagoya-u.jp

Institute for Space-Earth Environmental Research, Nagoya University, Aichi 464-8601, Japan whether the next solar cycle 25 will also show a weak activity such as the Maunder minimum or a relatively high activity comparable to solar cycle 22 . Thus far, various researchers have tried to study solar cycle prediction using various methods, although the results remain controversial. In recent years, significant attention has been paid to a method of predicting the next cycle activity by estimating the strength of a magnetic field within a polar region, which is considered a seed of the next solar cycle (Cameron and Schüssler 2015). The positive correlation between the intensity of the magnetic field within the polar region at the solar minimum and the activity during the next solar cycle has been confirmed in recent cycles. The surface flux transport (SFT) model has often been used to calculate the temporal evolution of the full magnetic field of the sun (e.g., Upton and Hathaway 2014; Cameron et al. 2016; Iijima et al. 2017, 2019), and several studies have succeeded in estimating the polar magnetic fields (Jiang et al. 2014). By contrast, the SFT model requires several 
parameters such as the meridional flow, differential rotation speed, and turbulent diffusion. These parameters have also been discussed based on observations (e.g., Hathaway and Rightmire 2011).

The solar surface rotates differentially in terms of latitude (e.g., Schroeter 1985), which is called differential rotation. The rotation is fastest near the equator and slower at high latitudes. At the equator, the sun rotates approximately once every 25 days, and it takes more than 30 days near the poles. The rotational speed of the sun is estimated using various methods. The rate of rotation has been studied using Doppler measurements at the solar surface (e.g., Ulrich et al. 1988). The solar differential rotation can also be determined from the positions of the observations of large numbers of magnetic features (e.g., Komm et al. 1993). The EUV and X-ray bright points, which are small-scale structures in the corona, have also been studied to estimate the differential rotation (e.g., Brajša et al. 2001). The EUV bright points have also been used in well-known studies of torsional oscillations and Rossby waves in the convection (e.g., McIntosh et al. 2019).

It is thought that there is a poleward flow from the equator to the pole on the surface, and there is an equatorward flow from the pole to the equator at the bottom of the convective layer, which is called meridional circulation (e.g., Hathaway and Upton 2014). Observational estimations of the poleward meridional flow in the photosphere have been conducted for numerous years by the tracking of the magnetic elements or surface Doppler signals from supergranulations. It is difficult to establish the basic characteristics of a meridional flow because the flow speed is $2-3$ orders of magnitude slower than the flow related to the solar rotation. It has also been discussed whether the meridional flow varies with time. The meridional flows may vary with the solar cycle because the presence of the sunspot disturbs the flows on the solar surface.

Thus far, numerous studies have been devoted to understanding the characteristics of differential rotation and meridional circulation. However, the solar cyclerelated variation of the solar surface flow remains unclear (e.g., Javaraiah and Ulrich 2006). Imada and Fujiyama (2018) recently claimed that the surface flow velocities estimated by the magnetic element feature tracking technique clearly depend on the average magnetic field strength of individual magnetic elements. The surface flow velocities in the solar cycle 24 might differ from the usual cycle because solar cycle 24 has been the weakest cycle during the last 100 years. In this study, we consider the temporal variation of solar differential rotation and poleward meridional flow during solar cycle 24 using the magnetic element feature tracking technique.

\section{Data and methods}

A series of line-of-sight magnetograms obtained using the helioseismic and magnetic imager (HMI) aboard the Solar Dynamics Observatory (SDO) (Scherrer et al. 2012) at a cadence of $1 \mathrm{~h}$ were used in this study. We analyzed the data from May 01, 2010 to March, 262020 (from 2096 to 2229 Carrington rotation), which corresponds approximately to the entire period of solar cycle 24 . The absolute values of the magnetograms were calibrated in the same way as described by Liu et al. (2012). We assumed that the line-of-sight magnetic field is largely radial, and we divided the magnetic field strength at each image pixel using the cosine of the heliographic angle from the disk center to minimize the apparent variations in field strength longitudinally from the central meridian. Using the equidistant cylindrical projection, we mapped each full-disk magnetogram onto heliographic coordinates (e.g., Komm et al. 1993; Hathaway and Rightmire 2011). The resolution of the projected map is $0.1^{\circ}$, and the range of the projection is $\pm 90^{\circ}$ for the central meridional distance (CMD) and latitude. We only used the distance from the center to less than $75^{\circ}$ to avoid noisy data. We also corrected the solar rotation axis in the same way as described by Hathaway and Rightmire (2011). Howard et al. (1984) and Beck and Giles (2005) found that the position of the sun's rotation axis to the heliographic coordinates is in error by $\sim 0^{\circ} .08$.

Numerous researchers have recently discussed solar surface flows using the magnetic element feature tracking technique (e.g., Berger et al. 1998; Hagenaar et al. 1999; Cadavid et al. 1999; Iida et al. 2012; Lamb 2017; Imada et al. 2020). We set a threshold of $40 \mathrm{G}$ for the magnetic field strength to pick up each magnetic element using a clumping method (Imada and Fujiyama 2018). The magnetic element features were selected when the total magnetic flux inside the magnetic element was larger than $10^{19} \mathrm{Mx}$. These threshold values were derived from an evaluation of the noise level in Michelson Doppler imager (MDI) on the Solar and Heliospheric Observatory (SOHO) (Scherrer et al. 1995; Parnell et al. 2009). We masked out the magnetic elements close to a sunspot $(<100 \mathrm{Mm})$ because it is well known that the magnetic elements near a sunspot behave differently (Komm et al. 1993). We defined the magnetic elements that had a total magnetic flux of larger than $10^{21} \mathrm{Mx}$ as a sunspot. We tracked the magnetic element motions after their detection. Because the rotation speed of the sun is $10-15 \mathrm{deg} /$ day, the moving distance of the magnetic element per hour is approximately $0.4-0.7^{\circ}$. Therefore, we identified the same magnetic elements between two images within $-0.1^{\circ}$ to $+1.0^{\circ}$ in the longitudinal direction and $-0.3^{\circ}$ to $+0.3^{\circ}$ in the latitudinal direction. In general, detecting the merging and splitting of the magnetic elements 
is difficult and results in uncertainties (Schrijver et al. 1997; Iida et al. 2012). To avoid these uncertainties, we tracked only the elements in which the total magnetic flux changes slightly $\left(0.1>\left|\log 10\left(\Phi_{2} / \Phi_{1}\right)\right|\right.$, where $\Phi_{1}$ and $\Phi_{2}$ are the magnetic flux of the magnetic elements before and after). When there were several candidates, we selected the element that had the lowest $\left|\log 10\left(\Phi_{2} / \Phi_{1}\right)\right|$ value. The method for detecting and tracking the magnetic elements is the same as that described by Imada and Fujiyama (2018). An example of the detection is shown in Fig. 1 of Imada and Fujiyama (2018), in which 309 magnetic element features were detected in one fulldisk magnetogram. In our study, we analyzed the data from May 01, 2010 to March, 26 2020, and totally we have detected more than $2.5 \times 10^{7}$ magnetic element features.

\section{Results \\ Differential rotation}

Figure 1 shows the solar cycle-related variations of the differential rotation during solar cycle 24 obtained using the magnetic element feature tracking technique. Figure 1a shows the temporal variation of the sunspot numbers as a reference (data are from the Royal Observatory of Belgium). Solar cycle 24 has had the weakest cycle during the last 100 years. During the rising phase of the cycle, from May 2010 to December 2011, as represented by the vertical dashed line in Fig. 1, the number of sunspots increased and reached the first peak, corresponding to the peak number of sunspots in the northern hemisphere. During the maximum phase of the cycle from January 2012 to December 2015, the number of sunspots reached the second peak in approximately the middle of 2014, which corresponds to the peak number of sunspots in the southern hemisphere. During the declining phase of the cycle from January 2015 to March 2020, the numbers of sunspots gradually and monotonically decreased with time, reaching almost zero in 2020. Sunspots for the new cycle, which have emerged at high latitudes, can often be observed these days (not shown here).

The temporal variations of the solar differential rotation speed profiles derived from the entire dataset from May 012010 to March 262020 are shown in Fig. 1b. The velocities are taken relative to the Carrington frame of reference, which has a sidereal rotation rate of 14.184 deg/day. A faster rotation is indicated by yellow, and a slower rotation is indicated by blue. The latitudinal centroid of the sunspot area in each hemisphere for each rotation is shown in red. We cannot observe any temporal variation in the profile shown in Fig. $1 \mathrm{~b}$.

Figure 1c shows the differences between the differential rotation profiles from the average. The average differential rotation profile was estimated by averaging the profiles of the entire dataset. Faster (prograde relative to the

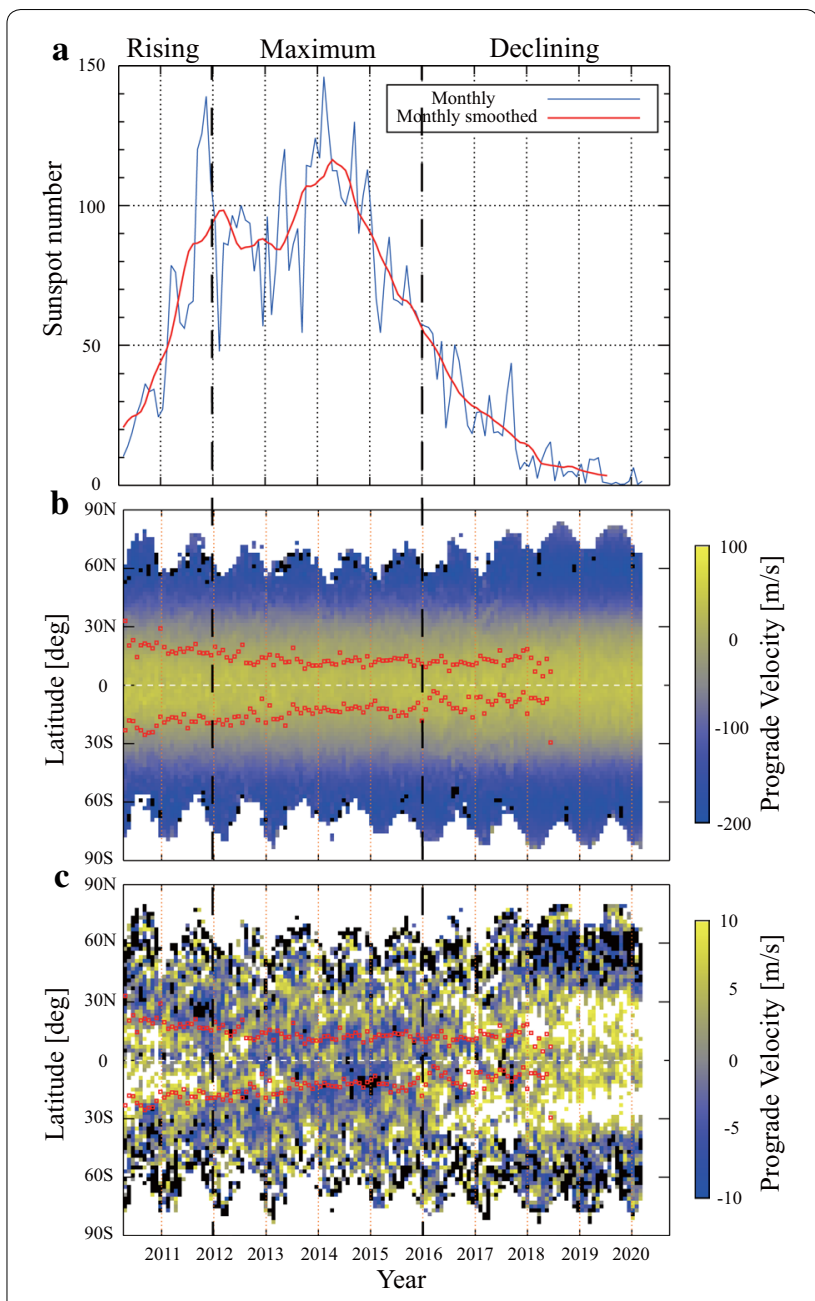

Fig. 1 Solar cycle-related variation of the differential rotation speed during solar cycle 24 obtained by the magnetic element feature tracking technique: a temporal variation of the sunspot numbers (data are from the Royal Observatory of Belgium), $\mathbf{b}$ temporal variation of the differential rotation speed, and $\mathbf{c}$ differences in the differential rotation profiles from the average are shown

average profile)/slower (retrograde) flows are indicated by yellow/blue. During the rising phase of the cycle, we can see the faster/slower flows on the equatorward/poleward sides of the sunspot area. The torsional oscillations (e.g., Howard and Labonte 1980) appear as a faster flow on the equatorward sides of the sunspot area and as a slower flow on the poleward sides. During the maximum phase of the cycle, the slower flow areas move from high latitudes to low latitudes associated with the movement of the sunspot area. Although not clear, faster flow areas that occur close to the pole can be seen during the maximum phase of the cycle. During the declining phase of the cycle, the faster flow areas move toward the equator, and the slower flow areas appear at high latitudes. 
Figure 2a shows the average differential rotation speed profile in the northern and southern hemispheres during the entire period of solar cycle 24 . The red/blue lines represent the northern/southern hemisphere results. For comparison, we also added the fitted curve of the differential rotation speed profile developed by Hathaway and Rightmire (2011), as shown by the black line. The uncertainties of the velocities can be evaluated from the standard deviations. The standard deviations are a few $\mathrm{m}$ $\mathrm{s}^{-1}$, which is negligibly small (not shown here, see Imada and Fujiyama (2018)). The differential rotation velocity range is $+30 /-180 \mathrm{~m} \mathrm{~s}^{-1}$ over a latitude range of $0 / 60^{\circ}$ in the Carrington rotation frame, respectively. As shown in Fig. 2a, the angular rotation rate is nearly identical to that found by Hathaway and Rightmire (2011) for solar cycle 23 using a different method (a cross-correlation technique). We can see a weak north-south asymmetry in solar cycle 24, which was previously reported by Imada and Fujiyama (2018). At high latitudes $\left(\sim 60^{\circ}\right)$, the rotational speed was slightly faster in the southern hemisphere than in the northern hemisphere.

Figure $2 b-d$ shows the average differential rotation speed profile during the rising, maximum, and declining phases of solar cycle 24, respectively. We cannot see any north-south asymmetry in Fig. 2b. The flattening of the profile at the equator, which has also been discussed in previous studies (Snodgrass 1983), can be seen during the rising phase of solar cycle 24. During the maximum phase of the cycle, we can clearly see that the rotation speeds in both hemispheres decelerate at mid-latitude $\left(\sim 20^{\circ}\right)$ and accelerate at high latitudes $\left(\sim 60^{\circ}\right)$, as shown in Fig. 2c. By contrast, we can see that the rotation speeds in both hemispheres accelerate at mid-latitudes and decelerate at high latitudes during the declining phase of the cycle, as shown in Fig. 2d.

\section{Meridional flow}

Figure 3 shows the solar cycle-related variations in the poleward meridional flow profile during solar cycle 24 obtained by the magnetic element feature tracking technique. Figure $3 \mathrm{a}$ is the same as Fig. 1a. Figure $3 \mathrm{~b}$ shows the temporal variations in the meridional flow profile derived from the entire dataset from May 1, 2010 to March 26, 2020. A poleward flow is indicated by yellow, and an equatorward flow is indicated by blue. The latitudinal centroid of the sunspot area in each hemisphere is shown in red. For a meridional flow, we can find two typical types of temporal variation of the profile in Fig. 3b. First, in the declining phase of the cycle, the meridional flow is accelerated in the middle latitude. Second, although not clear, equatorward flows at high latitudes $\left(\sim 60^{\circ}\right)$ occurred during approximately 2016-2018 (for
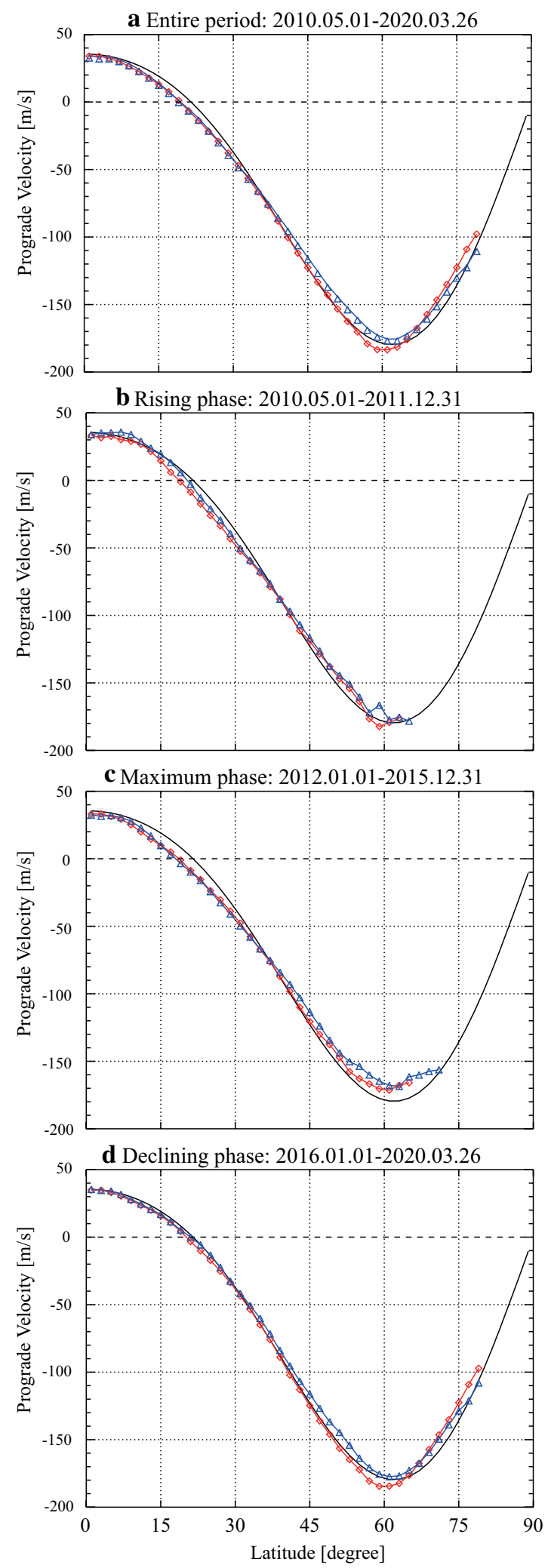

Fig. 2 Average differential rotation speed profile in the northern (red) and southern (southern) hemispheres a during the entire period, $\mathbf{b}$ rising, c maximum, and $\mathbf{d}$ declining phases of solar cycle 24 


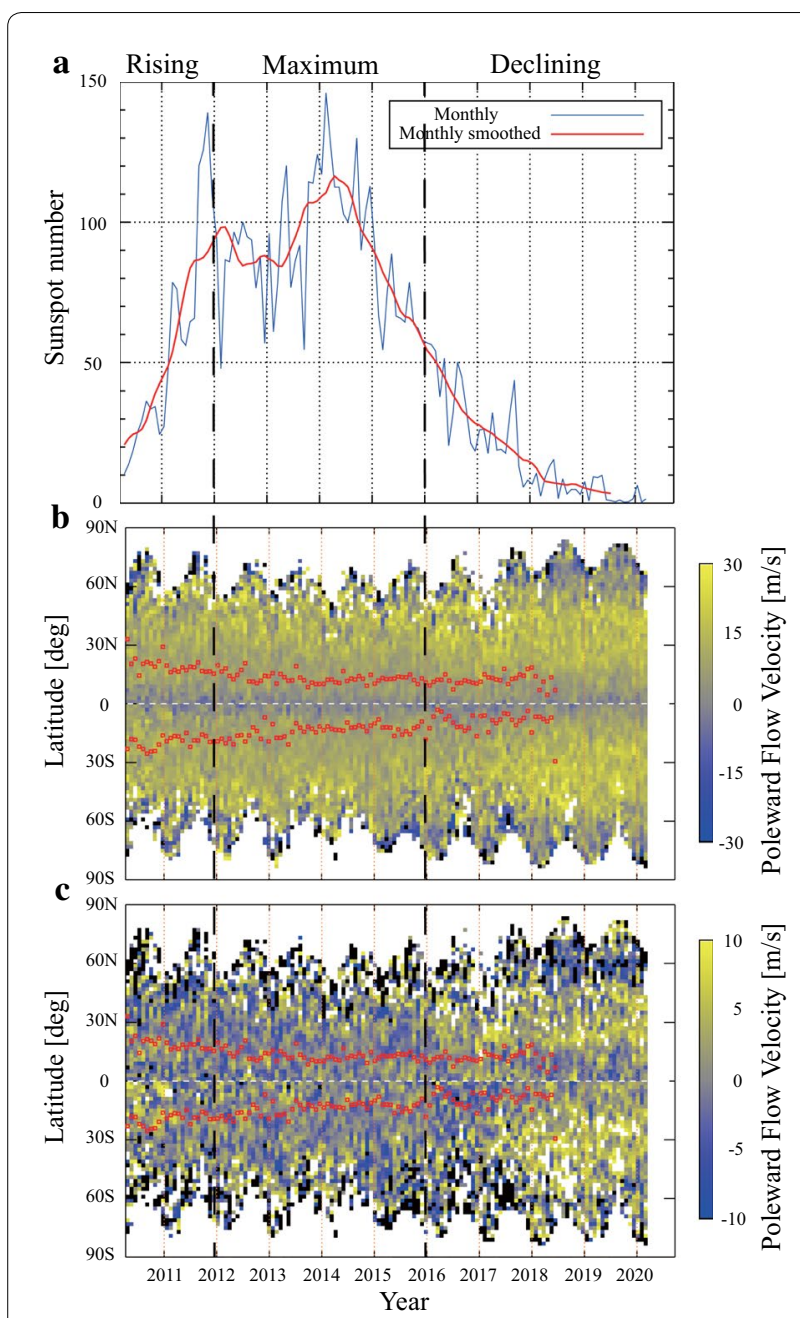

Fig. 3 Solar cycle-related variation of the poleward meridional flow during solar cycle 24 obtained using magnetic element feature tracking technique: a temporal variation of the sunspot numbers (data are from the Royal Observatory of Belgium), b temporal variation of the meridional flow, and $\mathbf{c}$ differences in the meridional flow from the average are shown

the southern hemisphere) and 2018-2020 (for the northern hemisphere).

Figure 3c shows the differences of the meridional flow profiles from the average. The average meridional flow profile was estimated by averaging the profiles of the entire dataset. Faster/slower poleward flows are indicated by yellow/blue. Although not as clear as a differential rotation, we can see the faster/slower flows on the equatorward/poleward sides of the sunspot area during the rising phase of the cycle. The faster flow areas that occur close to the pole can also be seen during the maximum phase, although faintly. During the declining phase of the cycle, the faster flow areas move toward the equator, and the slower flow areas appear at high latitudes. The faster area in the low latitudes appears first in the northern hemisphere at the beginning of the declining phase $(\sim 2016)$, and later also appears in the southern hemisphere $(\sim 2017)$. In the northern hemisphere, the slower flow area near the pole was more pronounced than in the southern hemisphere.

Figure 4a shows the bf average meridional flow profile in the northern and southern hemispheres during the entire period of solar cycle 24 . The red/blue lines represent the northern/southern hemisphere results. For comparison, we also added the fitted curve of the meridional flow profile developed by Hathaway and Rightmire (2011), as shown in the black line. The uncertainties of the velocities can be evaluated from the standard deviations. The standard deviations are a few $\mathrm{m} \mathrm{s}^{-1}$, which is still enough small. The meridional flow velocity profile peaked at $+15 \mathrm{~m} \mathrm{~s}^{-1}$ at $45^{\circ}$. As shown in Fig. 4a, the meridional flow in this study is faster/slower at low/high latitudes than that fitted by Hathaway and Rightmire (2011). We can see a weak north-south asymmetry in solar cycle 24 . At high latitude $\left(\sim 60^{\circ}\right)$, the poleward flow is slightly faster in the southern hemisphere than in the northern hemisphere.

Figure $4 \mathrm{~b}-\mathrm{d}$ shows the average meridional flow speed profile during the rising, maximum, and declining phases of solar cycle 24 , respectively. The meridional flow profile during the rising phase is nearly identical to that estimated by Hathaway and Rightmire (2011), although the meridional flow in the southern hemisphere is slightly faster than that in the northern hemisphere. During the maximum phase of the cycle, the flow of the northern hemisphere at high latitudes $\left(\sim 50^{\circ}\right)$ seems to be accelerated. The meridional flow of the middle latitude during the declining phase of the cycle was accelerated at up to $17 \mathrm{~m} \mathrm{~s}^{-1}$ in both hemispheres and decelerated at high latitudes $\left(>60^{\circ}\right)$.

\section{Summary and discussion}

We studied the solar cycle-related variation of the differential rotation using a magnetic element feature tracking technique using magnetic field data from May 1, 2010 to March 26, 2020 ( entire cycle of solar cycle 24). The average differential rotation velocity profile at a latitude of $0 / 60^{\circ}$ on the Carrington rotation frame was found to be approximately from 30 to $-180 \mathrm{~m} \mathrm{~s}^{-1}$. The profiles of the differential rotation velocity are mostly the same as those found by Hathaway and Rightmire (2011) for solar cycle 23 using different methods. Typical properties resulting from torsional oscillations can also be observed in solar cycle 24 . The observed amplitude of the variation is almost $\pm 10 \mathrm{~m} \mathrm{~s}^{-1}$. We also studied the solar cycle-related variation of the meridional flow. The average meridional 

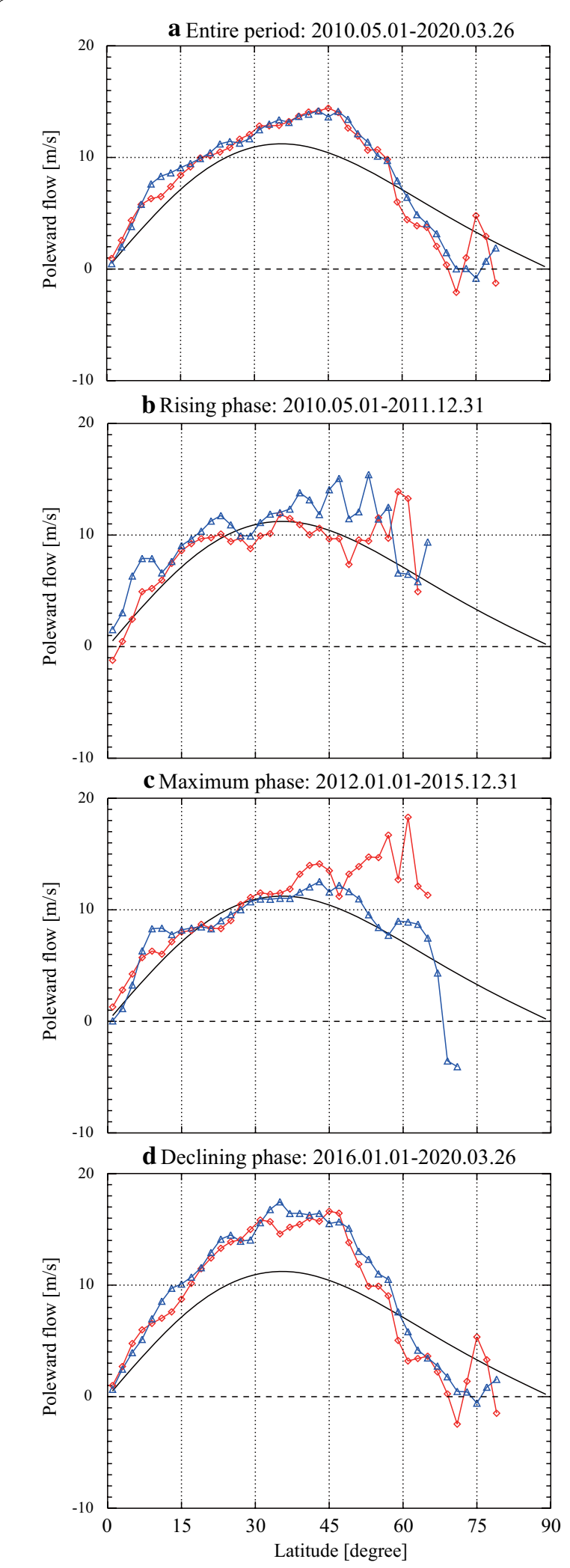

Fig. 4 Average poleward meridional flow profile in the northern (red) and southern (blue) hemispheres a during the entire period, $\mathbf{b}$ rising, c maximum, and $\mathbf{d}$ declining phases of solar cycle 24 flow profile reached up to $\sim 15 \mathrm{~m} \mathrm{~s}^{-1}$ at $45^{\circ}$. During the declining phase of the cycle, the meridional flow of the middle latitude $\left(30^{\circ}\right)$ is accelerated from 10 to $17 \mathrm{~m} \mathrm{~s}^{-1}$ in both hemispheres. One of our important findings is the meridional flow changes by up to $50 \%$ during the declining phase of solar cycle 24 . The temporal change of the meridian flow is similar to that of the differential rotation. The causal relationship between the torsional oscillation and the temporal variation of the meridian flow is not clear. Although the average meridional flow observed in solar cycle 24 is faster at middle latitudes (from $30^{\circ}$ to $60^{\circ}$ ) and slower at high latitude (from $60^{\circ}$ to $75^{\circ}$ ) than that observed in solar cycle 23 , our derived velocities are reasonable and consistent with past observations (e.g., Hathaway and Rightmire 2011).

Let us discuss the impact of our findings on the polar magnetic field estimation using the SFT model. The cross-equatorial transport of the net magnetic flux is important for estimating the polar magnetic field at the solar minimum (Cameron et al. 2013). The cross-equatorial transport of the net magnetic flux can be highly affected by the ratio between the meridional flow and turbulent diffusion. A pair of sunspots, on average, shows the leading sunspot closer to the equator than the following sunspot, which is known as Joy's law. If the ratio of meridional flow to the turbulent diffusion is larger, the cross-equatorial transport should be smaller, and vice versa. Therefore, the temporal variation of the meridional flow velocity is an important parameter for determining the polar magnetic field at the solar minimum. Although several systematic parameter studies of the influence of various parameters have been conducted on the polar magnetic field using the SFT model, most studies generally use a constant meridional flow velocity. By contrast, our results show that the meridional flow changes by almost $50 \%$ during the declining phase of the cycle. This variation might cause a significant reduction in the cross-equatorial transport of the net magnetic flux. It is important to continue to observe the temporal variation of meridional flow whether this trend hold true for solar cycle 25. Iijima et al. (2017) found that the crossequatorial flux transport is nearly zero during the declining phase of the cycle. This causes the newly emerged sunspots to not contribute to the variation in the axial dipole moment/polar magnetic field at the solar minimum, which is important for future solar cycle prediction. A quantitative analysis of the relationship between the poleward meridional flow and the cross-equatorial transport of the net magnetic flux is important for future studies.

Finally, we discuss the reason why the average poleward meridional flow observed in solar cycle 24 is faster at middle latitudes and slower at high latitude than that 
observed in solar cycle 23. Imada and Fujiyama (2018) found that magnetic elements with a strong or weak magnetic field show a slower/faster poleward meridional flow velocity. As previously mentioned, solar cycle 24 has been the weakest cycle during the last 100 years. Therefore, there is less magnetic flux on the solar surface, which might cause a faster meridional flow.

\section{Abbreviations}

SFT:: Surface flux transport; HMI:: Helioseismic and magnetic imager; SDO:: Solar Dynamics Observatory; MDI:: Michelson Doppler imager; SOHO:: Solar and Heliospheric Observatory.

\section{Acknowledgements}

We would like to thank the referees for useful comments that helped improving the manuscript. The authors thank K. Kusano, H. Hotta, and Y. lida for fruitful discussions. The Solar Dynamics Observatory is part of NASA's Living with a Star program.

\section{Authors' contributions}

SI analyzed the observation data and drafted the manuscript. KM and MF also analyzed the observation. All authors contributed to the interpretations of the data and the writing of the paper. All authors read and approved the final manuscript

\section{Funding}

This work was partially supported by the Grant-in-Aid for 17K14401 and 15H05816. This work was also partially supported by ISEE CICR International Workshop program, and the authors thank all members of the workshop. This work is supported by the NINS program for cross-disciplinary study on Turbulence, Transport, and Heating Dynamics in Laboratory and Solar/Astrophysical Plasmas: SoLaBo-X (Grant Numbers 01321802 and 01311904). A part of this study was carried out using the computational resources of the Center for Integrated Data Science, Institute for Space-Earth Environmental Research, Nagoya University.

\section{Availability of data and materials}

The SDO/HMI data are available from http://jsoc.stanford.edu. The sunspot number can be downloaded from http://www.sidc.be/silso/datafiles.

\section{Competing interests}

The authors declare that they have no competing interests.

Received: 12 May 2020 Accepted: 10 November 2020

Published online: 26 November 2020

\section{References}

Beck JG, Giles P (2005) Helioseismic determination of the solar rotation axis. Astrophys J Lett 621(2):L153-L156. https://doi.org/10.1086/429224

Berger TE, Löfdahl MG, Shine RA, Title AM (1998) Measurements of solar magnetic element dispersal. Astrophys J 506(1):439. https://doi. org/10.1086/306228

Brajša R, Wöhl H, Vršnak B, Ruždjak V, Clette F, Hochedez JF (2001) Solar differential rotation determined by tracing coronal bright points in SOHO-EIT images. I. Interactive and automatic methods of data reduction. Astron Astrophys 374:309-315. https://doi.org/10.1051/00046361:20010694

Cadavid AC, Lawrence JK, Ruzmaikin AA (1999) Anomalous diffusion of solar magnetic elements. Astrophys J 521(2):844. https://doi. org $/ 10.1086 / 307573$

Cameron R, Schüssler M (2015) The crucial role of surface magnetic fields for the solar dynamo. Science 347(6228):1333-1335. https://doi.org/10.1126/ science. 1261470
Cameron RH, Dasi-Espuig M, Jiang J, Işık E, Schmitt D, Schüssler M (2013) Limits to solar cycle predictability: cross-equatorial flux plumes. Astron Astrophys 557:A141. https://doi.org/10.1051/0004-6361/201321981

Cameron RH, Jiang J, Schüssler M (2016) Solar cycle 25: another moderate cycle? Astrophys J Lett 823(2):L22. https://doi. org/10.3847/2041-8205/823/2/L22

Clette F, Svalgaard L, Vaquero JM, Cliver EW (2014) Revisiting the sunspot number. A 400-year perspective on the solar cycle. Space Sci Rev 186(1-4):35103. https://doi.org/10.1007/s11214-014-0074-2

Hagenaar HJ, Schrijver CJ, Title AM, Shine RA (1999) Dispersal of magnetic flux in the quiet solar photosphere. Astrophys J 511(2):932. https://doi. org/10.1086/306691

Hathaway DH, Rightmire L (2011) Variations in the axisymmetric transport of magnetic elements on the sun: 1996-2010. Astrophys J 729(2):80. https:// doi.org/10.1088/0004-637X/729/2/80

Hathaway DH, Upton L (2014) The solar meridional circulation and sunspot cycle variability. J Geophys Res 119(5):3316-3324. https://doi. org/10.1002/2013JA019432

Howard R, Labonte BJ (1980) The sun is observed to be a torsional oscillator with a period of 11 years. Astrophys J Lett 239:L33-L36. https://doi. org/10.1086/183286

Howard R, Gilman PI, Gilman PA (1984) Rotation of the sun measured from Mount Wilson white-light images. Astrophys J 283:373-384. https://doi. org/10.1086/162315

lida Y, Hagenaar HJ, Yokoyama T (2012) Detection of flux emergence, splitting, merging, and cancellation of network field. I. Splitting and merging. Astrophys J 752(2):149. https://doi.org/10.1088/0004-637X/752/2/149

lijima H, Hotta H, Imada S, Kusano K, Shiota D (2017) Improvement of solarcycle prediction: plateau of solar axial dipole moment. Astron Astrophys 607:L2. https://doi.org/10.1051/0004-6361/201731813

lijima H, Hotta H, Imada S (2019) Effect of morphological asymmetry between leading and following sunspots on the prediction of solar cycle activity. Astrophys J 883(1):24. https://doi.org/10.3847/1538-4357/ab3b04

Imada S, Fujiyama M (2018) Effect of magnetic field strength on solar differential rotation and meridional circulation. Astrophys J Lett 864(1):L5. https:// doi.org/10.3847/2041-8213/aad904

Imada S, Hara H, Watanabe T, Kamio S, Asai A, Matsuzaki K, Harra LK, Mariska JT (2007) Discovery of a temperature-dependent upflow in the Plage region during a gradual phase of the X-class flare. PASJ 59:S793. https://doi. org/10.1093/pasj/59.sp3.S793

Imada S, Hara H, Watanabe T, Murakami I, Harra LK, Shimizu T, Zweibel EG (2011) One-dimensional modeling for temperature-dependent upflow in the dimming region observed by Hinode/EUV imaging spectrometer Astrophys J 743(1):57. https://doi.org/10.1088/0004-637X/743/1/57

Imada S, Aoki K, Hara H, Watanabe T, Harra LK, Shimizu T (2013) Evidence for hot fast flow above a solar flare arcade. Astrophys J Lett 776(1):L11. https ://doi.org/10.1088/2041-8205/776/1/L11

Imada S, Kato S, Fujiyama M (2020) Statistical analysis of asymmetric sunspot decay observed by Hinode. Solar Phys 295(1):154. https://doi. org/10.1007/s11207-020-01724-x

Javaraiah J, Ulrich RK (2006) Solar-cycle-related variations in the solar differential rotation and meridional flow: a comparison. Solar Phys 237(2):245265. https://doi.org/101007/s11207-006-0130-5

Jiang J, Hathaway DH, Cameron RH, Solanki SK, Gizon L, Upton L (2014) Magnetic flux transport at the solar surface. Space Sci Rev 186(1-4):491-523. https://doi.org/10.1007/s11214-014-0083-1

Komm RW, Howard RF, Harvey JW (1993) Rotation rates of small magnetic features from two-dimensional and one-dimensional cross-correlation analyses. Solar Phys 145(1):1-10. https://doi.org/10.1007/BF00627979

Lamb DA (2017) Measurements of solar differential rotation and meridional circulation from tracking of photospheric magnetic features. Astrophys J 836(1):10. https://doi.org/10.3847/1538-4357/836/1/10

Liu Y, Hoeksema JT, Scherrer PH, Schou J, Couvidat S, Bush RI, Duvall TL, Hayashi K, Sun X, Zhao X (2012) Comparison of line-of-sight magnetograms taken by the solar dynamics observatory/Helioseismic and Magnetic Imager and Solar and Heliospheric Observatory/Michelson Doppler Imager. Solar Phys 279(1):295-316. https://doi.org/10.1007/ s11207-012-9976-x

McIntosh SW, Leamon RJ, Egeland R, Dikpati M, Fan Y, Rempel M (2012) What the sudden death of solar cycles can tell us about the nature of the solar interior. Solar Phys 294(7):88. https://doi.org/10.1007/s11207-019-1474-y 
Parnell CE, DeForest CE, Hagenaar HJ, Johnston BA, Lamb DA, Welsch BT (2009) A power-law distribution of solar magnetic fields over more than five decades in flux. Astrophys J 698(1):75-82. https://doi. org/10.1088/0004-637X/698/1/75

Pesnell WD (2016) Predictions of solar cycle 24: How are we doing? Space Weather 14(1):10-21. https://doi.org/10.1002/2015SW001304

Scherrer PH, Bogart RS, Bush RI, Hoeksema JT, Kosovichev AG, Schou J, Rosenberg W, Springer L, Tarbell TD, Title A, Wolfson CJ, Zayer I, MDI Engineering Team (1995) The solar oscillations investigation-Michelson Doppler Imager. Solar Phys 162(1--2):129-188. https://doi.org/10.1007/BF007 33429

Scherrer PH, Schou J, Bush RI, Kosovichev AG, Bogart RS, Hoeksema JT, Liu Y, Duvall TL, Zhao J, Title AM, Schrijver CJ, Tarbell TD, Tomczyk S (2012) The Helioseismic and Magnetic Imager (HMI) Investigation for the Solar Dynamics Observatory (SDO). Solar Phys 275(1-2):207-227. https://doi. org/10.1007/s11207-011-9834-2

Schrijver CJ, Title AM, van Ballegooijen AA, Hagenaar HJ, Shine RA (1997) Sustaining the Quiet Photospheric Network: the balance of flux emergence, fragmentation, merging, and cancellation. Astrophys J 487(1):424-436. https://doi.org/10.1086/304581

Schroeter EH (1985) The solar differential rotation-present status of observations. Solar Phys 100:141. https://doi.org/10.1007/BF00158426
Snodgrass HB (1983) Magnetic rotation of the solar photosphere. Astrophys J 270:288-299. https://doi.org/10.1086/161121

Svalgaard L, Cliver EW, Kamide Y (2005) Sunspot Cycle 24: Smallest Cycle in 100 Years? In: Sankarasubramanian K, Penn M, Pevtsov A (eds) Large-scale structures and their role in solar activity, astronomical society of the Pacific conference series, vol 346, p 401

Tsuneta S, Hara H, Shimizu T, Acton LW, Strong KT, Hudson HS, Ogawara Y (1992) Observation of a solar flare at the limb with the $\mathrm{YOHKOH}$ soft X-ray telescope. PASJ 44:L63-L69

Ulrich RK, Boyden JE, Webster L, Snodgrass HB, Padilla SP, Gilman P, Shieber T (1988) Solar rotation measurements at MT.WILSON_part five. Solar Phys 117(2):291-328. https://doi.org/10.1007/BF00147250

Upton L, Hathaway DH (2014) Predicting the sun's polar magnetic fields with a surface flux transport model. Astrophys J 780(1):5. https://doi. org/10.1088/0004-637X/780/1/5

\section{Publisher's Note}

Springer Nature remains neutral with regard to jurisdictional claims in published maps and institutional affiliations.

\section{Submit your manuscript to a SpringerOpen ${ }^{\circ}$ journal and benefit from:}

- Convenient online submission

- Rigorous peer review

- Open access: articles freely available online

- High visibility within the field

- Retaining the copyright to your article

Submit your next manuscript at $\boldsymbol{\nabla}$ springeropen.com 